\title{
CONVECTIVE PROCESSES IN HIGH RESOLUTION MODELS: IMPACT OF THE LEAD TIME OF THE SIMULATION
}

\author{
Álvaro Lavín Gullón ${ }^{(1)}$, Jesús Fernández Fernández ${ }^{(2)}$, Rita M. Cardoso ${ }^{(8)}$, \\ Klaus Goergen $^{(5,6)}$, Sebastian Knist ${ }^{(7)}$, Torge Lorenz ${ }^{(9)}$, Josipa Milovac ${ }^{(4)}$, \\ Pedro M. M. Soares ${ }^{(8)}$, Stefan Sobolowski ${ }^{(9)}$, Heimo Truhetz ${ }^{(3)}$, \\ Kirsten Warrach-Sagi ${ }^{(4)}$ \\ (1) Santander Meteorology Group, Instituto de Física de Cantabria, \\ CSIC-Universidad de Cantabria, Santander, Spain, alvaro.lavin@unican.es \\ (2) Santander Meteorology Group, Dpto. de Matemática Aplicada y Ciencias de la Computación, \\ Universidad de Cantabria, Santander, Spain \\ (3) Wegener Center for Climate and Global Change, University of Graz, Austria \\ (4) Institute of Physics and Meteorology, University of Hohenheim, Stuttgart, Germany \\ ${ }^{(5)}$ Institute of Bio- and Geosciences (IBG-3), Research Centre Jülich, Jülich, Germany \\ ${ }^{(6)}$ Centre for High-Performance Scientific Computing in Terrestrial Systems, \\ Geoverbund ABC/J, Jülich, Germany \\ (7) Meteorological Institute, University of Bonn, Bonn, Germany \\ ${ }^{(8)}$ Instituto Dom Luiz (IDL), Faculdade de Ciências, Universidade de Lisboa, Portugal \\ ${ }^{(9)}$ Uni Research Climate, Bjerknes Centre for Climate Research, Bergen, Norway
}

\section{INTRODUCTION}

Most heavy precipitation events occurring in the world are associated with convective processes. As these phenomena produce severe economic and societal impacts, it is crucial to get to know their behaviour and their evolution in a future climate. For this reason, the international project CORDEX (Coordinated Regional climate Downscaling Experiment) proposed the Flagship Pilot Study on Convective phenomena at high resolution over Europe and the Mediterranean (FPS-CEM, Coppola et al., 2018), focused on the study of convection in Europe. In this initiative, multi-model and multi-physics results and uncertainties of regional climate models (RCMs) are explored by means of ensembles of simulations. In this work, we additionally explore the role of internal variability to explain the differences found in the results by different model configurations.

A few international initiatives have studied climate change through regional downscaling. One example is CORDEX (http://cordex.org), which aims at coordinating and advancing the science and the application of regional climate downscaling. In order to address more targeted scientific problems related to regional climate change, different Flagship Pilot Studies (FPS) were recently proposed within CORDEX. Among them, the FPS on convective phenomena at high resolution over Europe and the Mediterranean (Coppola et al., 2018) aims to improve the representation and future projections of these phenomena by reaching the so-called convection-permitting resolution (horizontal scale below $4 \mathrm{~km}$ ). Up to now, a few studies have evaluated the added value of solving convection explicitly in climate simulations (Prein et al., 2015). Three objectives were established: (1) To evaluate the effect of the climate change on convective processes and their impacts, (2) to identify possible improvements by using models which solve convection explicitly, as compared with lower resolution models, and (3) to identify the added value by using statistical models which mimic these models ("emulators"). Three phases are planned in FPS-CEM. The first phase, which is ongoing, aims at addressing 
the capability of models to reproduce convection in short-term runs (from days to 1 month), as not all RCMs involved have simulated at convection-permitting scales. The second phase will assess the capability of models to simulate present climate through 15 -year evaluation runs. Finally, the third phase will analyze convection in future climate scenarios by running time slices nested into global CMIP scenario runs.

A multi-model and multi-configuration ensemble was created among all groups participating in FPS-CEM. The full ensemble is composed of 22 members with different configurations of 6 RCMs. The Weather Research \& Forecasting (WRF; Skamarock et al., 2008) model is one of the contributing models, and has the advantage of offering a wealth of physical parameterization options. Therefore, all groups contributing with this model to the FPS-CEM have coordinated a multi-physics ensemble to explore the uncertainty associated to parameterizations. The target area of FPS-CEM is the Alps (Figure 1) due to the amount and quality of observational datasets available for model evaluation, and the importance of convective processes in the area. The minimum domain is centered over this region and its horizontal resolution was coordinated to be $\sim 3 \mathrm{~km}$. This domain (ALP3 ) is nested in a pan-european domain (EUR-11, 12 km), using 50 vertical levels. For the first phase, an experiment was carried out on several heavy precipitation events. These events were simulated in two modes: "weather-like" (WL) and "climate mode" (CM). WL mode is a short simulation, as those in numerical weather prediction, initialized a few days before the event. Initial conditions are a source of predictability, so this mode makes the best-case scenario to reproduce the observed event. This mode was compared to the "climate mode", which is a longer-term simulation mimicking standard climate simulations, initialized one month before the event. In this mode, initial conditions are not a source of predictability, but of internal variability. The observational information to reproduce the events is provided through the (distant) lateral boundaries of the RCM, which is relatively free to develop its own weather trajectory.

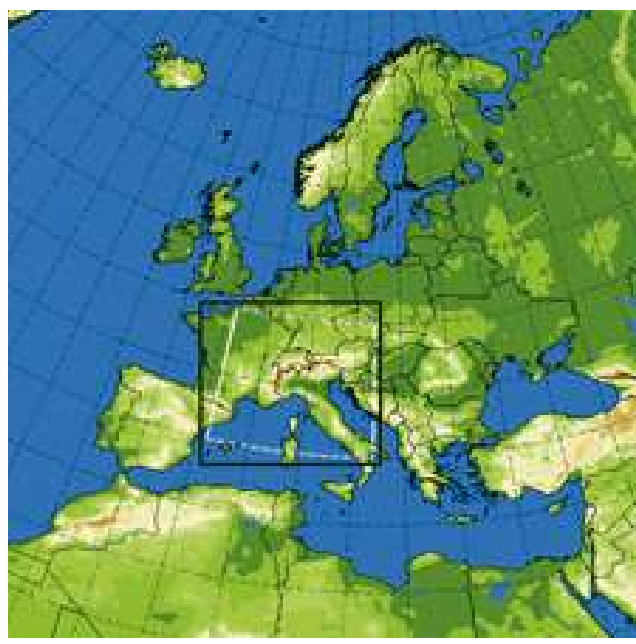

Three heavy precipitation events during fall and summer were selected. The first case (hereafter "IOP") occurred in October 2012, when a mesoscale convective system favored by a depression over the Gulf of Lion affected southern France and northern Italy. The second case (hereafter "Austria") is a convective orographic

Fig. 1. Domains in latitude-longitude rotated pole projection used by the WRF community for CM vs WL experiment. The pan-european (EUR-11, red) and alpine (ALP-3, black) domains have horizontal resolutions of $\sim 12$ and $\sim 3 \mathrm{~km}$, respectively. The minimum alpine domain (regular in latitude-longitude) is depicted in white. 
precipitation occurred in June 2009, when an isolated cutoff low induced a persistent northeasterly flow of warm-moist air on the austrian Alps. The third case (hereafter "Foehn") is a foehn event in November 2014, when a deep trough induced a persistent southerly flow over the Alps.

The results of these experiments regarding precipitation (ALP-3 domain) were very dependent on the case, the simulation mode, and the model (Coppola et al., 2018). In the IOP case, both modes showed large spatial and temporal differences between members over areas where precipitation is more influenced by the orography. Over these regions, a spread in WL becomes as noticeable as in CM. However, the ensemble mean was consistent in both modes, as compared to observations. The foehn case showed the best model performance. This is likely due to the strong synoptic forcing. Precipitation was well captured spatially and temporally when compared with observations. Besides, both $\mathrm{WL}$ and CM modes revealed good agreement. The Austria case was the most striking, as individual members showed a large spread with a wide range of model behaviours in $\mathrm{CM}$. The ensemble mean was close to the observations in WL, since most members reproduced the spatial pattern of precipitation, although with varying maxima. However, the ensemble mean in CM showed a strong underestimation due to the fact that some models captured the event well whereas others missed it completely.

The above experimental setup is intended to link the reproduction of particular extreme weather events to different parameterization sets. In particular, many configurations differ just in a single parameterization option. Therefore, in principle, the different results could be explained by a given parameterization choice for a given process. However, the experimental setup does not consider the sensitivity to initial conditions, also referred to as the RCM internal variability. Models are very sensitive to perturbations due to the chaotic behaviour of certain natural processes. This intrinsic factor in model simulations can be very significant (Giorgi et al, 2000; Christensen et al., 2001). Previous studies have analyzed the role of the internal variability showing that its influence depends on the season, with higher variability in summer (Caya et al., 2004). This indicates that boundary forcing data are not able to overcome the internal variability in certain atmospheric situations, when the atmospheric circulation is weaker. In contrast, Lucas-Picher et al. (2008) found some discrepancies by using a larger domain. An increased domain enhanced the internal variability in winter due to a lesser control of the driving fields. Laux et al. (2016) investigated, in a sensitivity study to land use changes, the number of ensemble members required to achieve confidence above the internal RCM variability. They found that at least five members are enough to derive acceptable results. This is in agreement with previous investigations which revealed that generally six members provide robustness (Lucas-Picher et al., 2008) .

In this work, we explore the uncertainty associated to the model internal variability as compared to the uncertainty arising from the use of different parameterization sets. We aim at distinguishing both sources of uncertainty, quantifying their relative size and determining whether the different results can be robustly linked to the use of particular parameterization sets. 


\section{METHODOLOGY AND DATA}

In this work we focus on a few members of the WRF model ensemble. All were produced with the Advanced Research WRF core, version 3.8.1. The ensemble considers different parameterizations for cloud microphysical processes, planetary boundary layer, surface and land processes, as well as radiative processes. As an example, the simulation from Universidad de Cantabria (UCAN) used the Double-Moment 6-class microphysics scheme, Mellor-Yamada Nakanishi and Niino planetary boundary layer scheme, Global/ Regional Integrated Modeling System shallow cumulus scheme, Revised MM5 MoninObukhov and NOAH-MP surface schemes, and RRTMG radiation scheme. Lateral boundary forcing and initial conditions are taken from ERA-Interim (Dee et al. 2011) reanalysis data at $0.75^{\circ} \times 0.75^{\circ}$ horizontal resolution.

In order to explore the role of internal variability, a multi-initial-condition ensemble was built following the lagged method (see e.g. Laux et al., 2016). It consists in generating different initial conditions by shifting the start date of the simulation. In our case, we shifted back, in 1-day steps, the start date of the standard UCAN simulation (hereafter UCAN-r0). Thus, three additional simulations were run by shifting 1 day (UCAN-r1), 2 days (UCAN-r2) and 3 days (UCAN-r3).

We focus on the large scale circulation in the intermediate domain (EUR-11, Figure 1), as represented by the geopotential height at $850 \mathrm{hPa}$. Model performance is evaluated by means of the Root-Mean Squared Error (RMSE) with respect to the ERA-Interim reanalysis, which provides an estimation of how the RCM simulations represent the observed circulation. The RMSE spread provides an indication of the uncertainty associated to the different ensembles (multi-model and multi-IC).

\section{RESULTS AND DISCUSSION}

In order to understand the behaviour of each member in the multi-physics ensemble, the large-scale circulation in CM, for a particular day (June 23rd, 2009) during the Austria event, was compared with that of ERA-Interim reanalysis (Figure 2). The large-scale circulation in WRF model simulation reproducing well the precipitation event (BCCR, as shown by Coppola et al., 2018) is consistent with reanalysis. Especially, the low pressure system centered over Croatia. However, other model configurations (IDL, FZJ-IBG3, FZJ-IGB3-2 or UCAN-r0, the latter can be seen in Fig. 5) simulate a low pressure system in southern Italy moving easterly, which alters the circulation so that the event over the Alps is missed.

Figure 3 depicts the temporal evolution of the $850 \mathrm{hPa}$ geopotential height RMSE with respect to reanalysis. In WL mode, RMSE values are low during most of the event, since they are still increasing from the very low initial values corresponding to the initial conditions taken from reanalysis. By the end of the event, the ensemble mean of the RMSE is about $20 \mathrm{~m}$. All members present the same behaviour, almost similar at the beginning and with a little spread (range $\sim 10 \mathrm{~m}$ ) by the end of the period. In this mode, initial conditions play a role as a source of predictability, since the event starts to develop only one day after the simulation start. This constrains the model, since the circulation is more controlled by the initial conditions, and hence the model is less free to simulate it. 

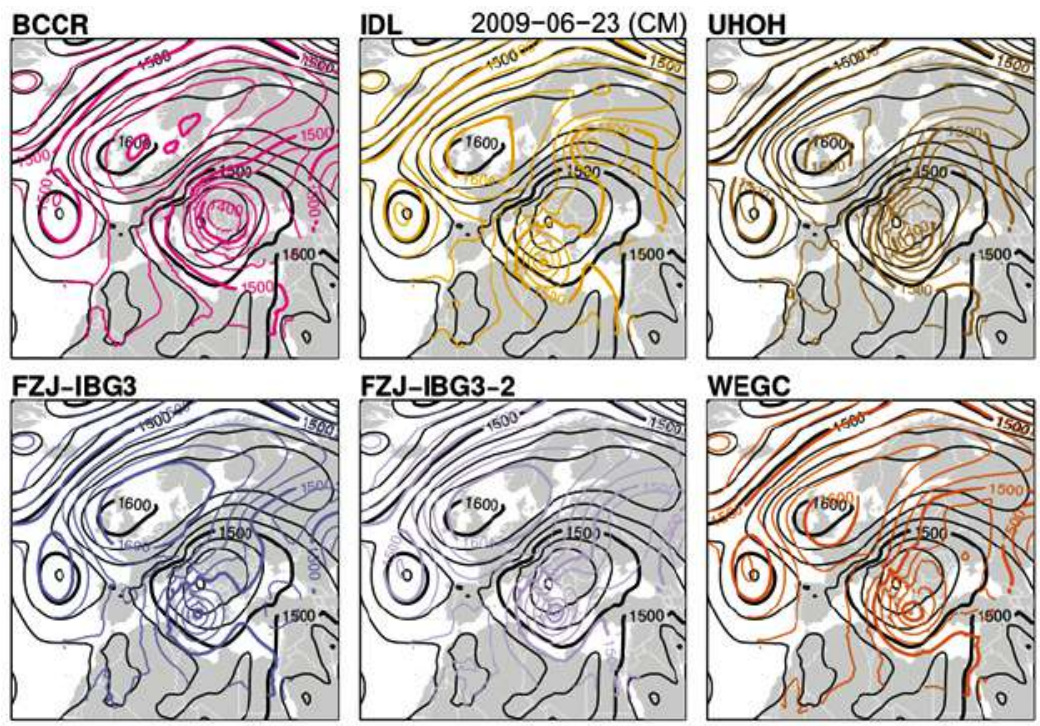

Fig. 2. Mean geopotential $850 \mathrm{hPa}$ fields (color lines) as compared with that from ERA-Interim reanalysis (black lines) on 23 June 2009, for a set of 6 WRF model physical parameterization configurations. Contour intervals are $25 \mathrm{~m}$.

In CM, however, the event is 20 days ahead of the simulation start, well beyond any predictability induced by initial conditions. The initially growing RMSE stabilizes at about $25 \mathrm{~m}$ after 5-6 days. After this time, any potential to reproduce an observed event must come through the lateral boundary forcing, which is taken from ERA-Interim every 6 hours. Therefore, during the event, initial conditions behave as a source of internal variability. Moreover, there are two RMSE peaks initiating around 12th and 17th June, when RMSE values reach up to $60 \mathrm{~m}$. This peaks are due to circulation discrepan-

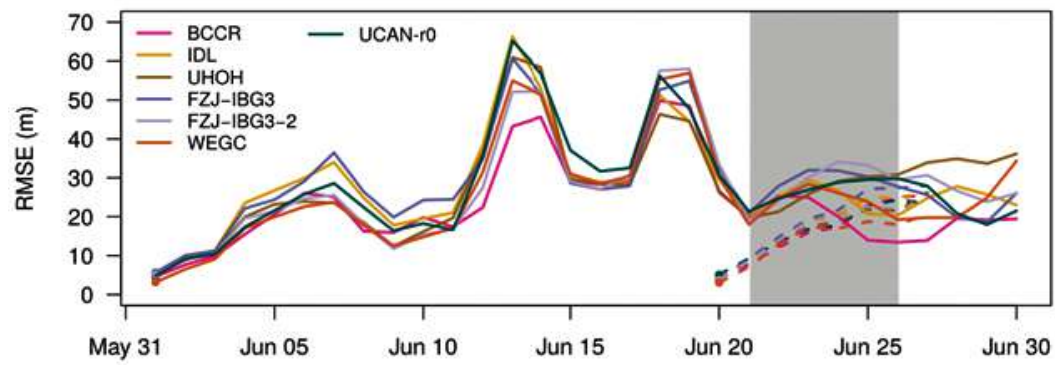

Fig. 3. RMSE (m) time series for geopotential height at $850 \mathrm{hPa}$ in all points of EUR-11 domain versus the same from ERA-Interim reanalysis. Solid (dashed) lines represent CM "climate mode"

(WL "weather-like"). The gray band shows the time period of the "Austria" event. 
cies with respect to reanalysis appearing in the northern side of the domain (Arctic area), where the forcing from the driving fields is weaker (Rinke et al., 2000). The flow, driven by the boundary conditions, comes back to an RMSE $\sim 25 \mathrm{~m}$ after these weak forcing episodes and keeps these values during the Austria event. The maximum inter-member (i.e. multi-physics) spread is $\sim 20 \mathrm{~m}$ during the event. This is noticeably larger than the spread in WL mode $(\sim 10 \mathrm{~m})$.

The spread of the ensemble with perturbed initial conditions (Figure 4, green lines) is as large as that of the multi-physics ensemble (grey lines). RMSE values are generally in the range of $15-35 \mathrm{~m}$; UCAN-r3 presents a $\sim 60 \mathrm{~m}$ peak by the end of the target event, produced by a non-observed low pressure system over Scandinavia. We can also see that slightly different initial conditions in a single WRF model configuration (Figure 5) reproduce a wealth of synoptic circulation conditions, similar to that in a multi-physics ensemble (cf. Figure 2). In particular, there are members reproducing the observed circulation (UCAN-r1), whereas others miss the event by simulating again the low pressure system in southern Italy (UCAN-r0, UCAN-r2, UCAN-r3), shown by other model configurations. This suggests that the spread appearing in the multi-physics ensemble could not only be explained because of different model configurations, but also by the model internal variability. For example, the model configurations run by BCCR and FZJ-IGB3-2 differ only in their land surface model (NOAH-MP and NOAH, respectively). One could argue that the

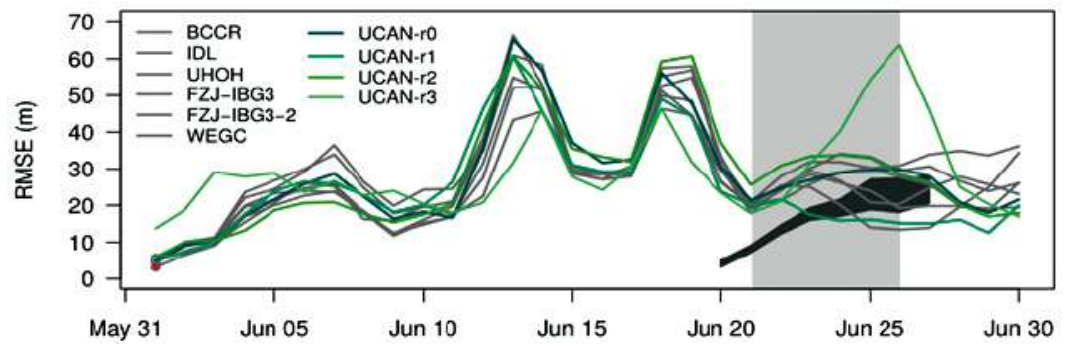

Fig 4. As Fig. 3, but including the multi-initial-condition ensemble (green lines). For clarity, the multiphysics CM ensemble has been greyed out and the WL range is represented by a dark grey shade.
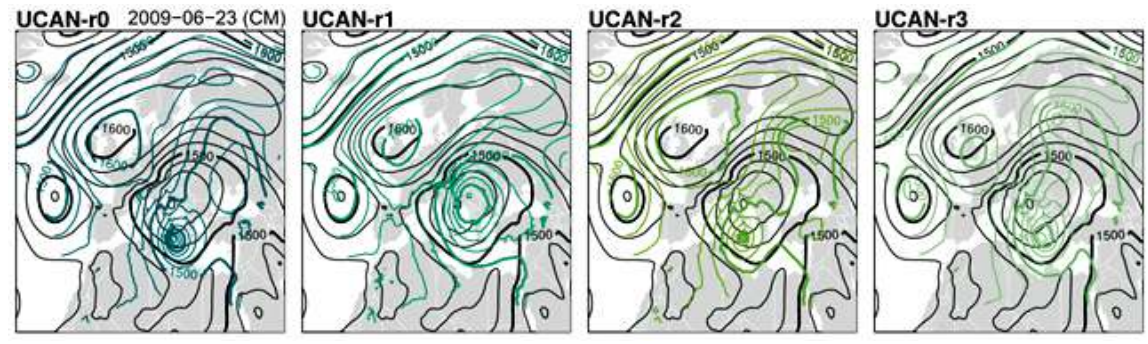

Fig 5. As Fig. 2, but for the UCAN multi-initial-conditions ensemble members. 
older NOAH scheme produces some particular change that triggers the low pressure system evolving along southern Italy. However, the same feature develops in other model configurations and can even appear just changing slightly the forecast lead time, as was done in the multi-initial-conditions ensemble. The system seems to have two preferred weather regimes and small changes in the trajectory (induced either by changes in the model configuration or by perturbing the initial conditions) make the system follow one or the other.

\section{CONCLUSIONS}

We compared the ability of a set of WRF model simulations to reproduce the large scale circulation leading to a heavy precipitation event over Austria. The simulations can be grouped according to their lead time with respect to the onset of the event: The WL experiment consists of a multi-physics ensemble initialized one day before the event, the CM experiment was started 20 days before, and a new set of simulations was included to account for the internal variability, with lead times ranging from 20 to 23 days, but using a single model configuration.

The spread of the WL experiment RMSE is smaller than that of the CM. However, by the end of the target event, there are ensemble members of the CM experiment resembling the observed circulation closer than any of the WL members. The singlemodel multi-initial-conditions ensemble shows similar or larger spread than the multi-physics CM ensemble. Moreover, similar synoptic circulation patterns were developed by the multi-physics and the internal variability ensemble. Therefore, caution should be taken when interpreting physical parameterization sensitivity studies, especially if spanning short testing periods as in this example. Whenever possible, RCM internal variability should be quantified to discern the effect of model modifications from the chaotic amplification of small perturbations.

This work is based on very preliminary results from the CORDEX FPS-CEM. Further work is ongoing to consider other events, longer simulations, multi-model ensembles, and to expand the ensemble to explore internal variability.

\section{ACKNOWLEDGEMENTS}

This work is funded by the Spanish government through grant BES-2016-078158 and MINECO/FEDER co-funded projects INSIGNIA (CGL2016-79210-R) and MULTI-SDM (CGL2015-66583-R). UCAN simulations have been carried out on the Altamira Supercomputer at the Instituto de Física de Cantabria (IFCA, CSIC-UC), member of the Spanish Supercomputing Network. UHOH simulations have been carried out at the supercomputing center HLRS in Stuttgart, Germany, supported by the German Science Foundation (DFG) through project FOR 1695. The authors gratefully acknowledge the computing time granted by the John von Neumann Institute for Computing (NIC) and provided on the supercomputer JURECA at Jülich Supercomputing Centre (JSC) under compute time project hka19. 


\section{References}

- Caya, D., Biner, S., 2004. Internal variability of RCM simulations over an annual cycle. Climate Dynamics. 22:33-46.

- Christensen, O.B, Gaertner, M.A., Prego, J.A, Polcher, J. 2001. Internal variability of regional climate models. Climate Dynamics. 17: 875-887.

- Coppola, E., Sobolowski, S., Pichelli, E., Raffaele F., Ahrens, B., Anders, I., Ban, N., Bastin, S., Belda, M., Belusic, et al., 2018. A first-of-its-kind multi-model convection permitting ensemble for investigating convective phenomena over Europe and the Mediterranean. Climate Dynamics. Submitted.

- Dee, D. P., Uppala, S. M., Simmons, A. J., Berrisford, P., Poli, P., Kobayashi, S., Andrae, U., Balmaseda, M. A., Balsamo, G., Bauer, P., et al., 2011. The ERA-Interim reanalysis: configuration and performance of the data assimilation system. Royal Meteorological Society. 137: 553-597.

- Giorgi, F., Bi, X., 2000. A study of internal variability of a regional climate model. Journal of Geophysical Research. 105: 29.503-29.521.

- Laux, P., Nguyen, P., Cullmann, J., Van, T. P., Kunstmann, H., 2017. How many RCM ensemble members provide confidence in the impact of land-use land cover change?. International Journal of Climatology. 37:2080-2100.

- Lucas-Picher, P., Caya, D., de Elía, R., Laprise, R., 2008. Investigation of regional climate models' internal variability with a ten-member ensemble of 10-year simulation over a large domain. Climate Dynamics. 31:927-940.

- Prein, A. F., Langhans, W., Fosser, G., Ferrone, A., Ban, N., Goergen, K., Keller, M., Tölle, M., Gutjahr, O., Feser, F., et al., 2015. A review on regional convection-permitting climate modeling: Demonstrations, prospects, and challenges. Rev. Geophys. 53:323-361.

- Rinke, A., Dethloff K., 2000. On the sensitivity of a regional Arctic climate model to initial and boundary conditions. Climate Research. 14:101-113.

- Skamarock, W. C., Klemp, J. B., Dudhia, J., Gill, D. O., Barker, D. M., Duda, M. G, Huang, X.-Y., Wang, W., and Powers, J. G. , 2008. A Description of the Advanced Research WRF Version 3. NCAR Tech. Note NCAR/TN-475+STR, 113. 